\title{
Caracterização das violências autoprovocadas cometidas pelas pessoas idosas na Região Sul do Brasil de 2009 a 2016
}

\author{
Characterization of self-inflicted violence committed by older people in southern Brazil from 2009 \\ to 2016
}

Fernanda Cornelius Langel $\mathbb{1 D}$ Carolina Carvalho Bolsoni? $\mathbb{D}$ Sheila Rubia Lindner' $\mathbb{D}$

\section{Resumo}

Objetivo: Descrever as características das pessoas idosas que cometeram violência autoprovocada, notificados no Sistema de Informação de Agravos de Notificação (SINAN) na região Sul do Brasil, de 2009 a 2016. Método: Trata-se de um estudo de abordagem quantitativa, retrospectivo, descritivo e com dados secundários. As variáveis em relação as características sociodemográficas dos locais e dos meios de agressão foram selecionadas com base nas fichas de notificação. As variáveis foram submetidas à análise estatística descritiva por meio de frequência simples e proporção (\%), foi estratificado por faixa etária (60-69 anos; 70-79 anos; 80 anos ou mais) e realizado os intervalos de confiança (IC95\%). A significância estatística foi testada através do teste qui-quadrado $\left(\chi^{2}\right)$ e considerado o valor de $p \leq 0,05$. Resultados: Os resultados mostraram que, na região Sul do Brasil, o perfil das pessoas idosas que cometeram violência autoprovocada são predominantemente de 60 a 69 anos (61,3\%), do sexo masculino (56,1\%), cor de pele branca $(90,9 \%)$, com baixo nível educacional (56,3\%) e casadas (54,0\%). Entre os estados, o Rio Grande do Sul registrou o maior número de notificações (50,7\%), predominaram como local de ocorrência a zona urbana $(81,8 \%$ ) e a residência/habitação coletiva $(90,2 \%)$. Os meios de agressão mais utilizados foram o enforcamento $(29,9 \%)$ e o envenenamento $(24,9 \%)$. Observou-se a ocorrência de dois desfechos, a repetição $(31,5 \%)$ da violência autoprovocada e os registros de óbitos (43,8\%). Conclusão: O delineamento do perfil epidemiológico, na região Sul do Brasil, identificou grupos de pessoas idosas que necessitam de maior atenção nas ações de prevenção e ocorrência da violência autoprovocada, sendo eles os idosos do sexo masculino, mais jovens e com baixa escolaridade.

\footnotetext{
Universidade Federal de Santa Catarina, Departamento de Saúde Pública, Programa de Pós-Graduação em Saúde Coletiva. Santa Catarina, SC, Brasil.
}

Financiamento da pesquisa: Coordenação de Aperfeiçoamento de Pessoal de Nível Superior - Brasil (CAPES). $\mathrm{N}^{\text {o }}$ do processo: 88882.437675/2019- 01. Bolsa de Mestrado.

Os autores declaram não haver conflito na concepção deste trabalho.

Palavras-chave: Violência. Tentativa de Suicídio-Idoso. Sistemas de Informação em Saúde. 


\section{Abstract}

Objective: To describe the characteristics of older people who committed self-inflicted violence, reported in the Notifiable Diseases Information System (SINAN) in southern Brazil, from 2009 to 2016. Method: This is a retrospective, descriptive study with a quantitative approach, and with secondary data. The variables in relation to the sociodemographic characteristics of the places and means of violence were selected based on the notification forms. Variables were subjected to descriptive statistical analysis using simple frequency and proportion (\%), stratified by age group (60-69 years; 70-79 years; 80 years or more) and confidence intervals were performed $(95 \% \mathrm{CI})$. Statistical significance was tested using the chi-square test $\left(\chi^{2}\right)$ and considered the value of $p \leq 0.05$. Results: The results showed that, in the southern region of Brazil, the profile of older people who committed self-inflicted violence are predominantly aged 60 to 69 years $(61.3 \%)$, male $(56.1 \%)$, white $(90.9 \%)$, with low educational level $(56.3 \%)$ and married $(54.0 \%)$. Among the states, Rio Grande do Sul recorded the highest number of notifications $(50.7 \%)$, urban areas $(81.8 \%)$ and residences/collective housing $(90.2 \%)$ predominated as places of occurrence. The most used means of violence were hanging $(29.9 \%)$ and poisoning $(24.9 \%)$. The occurrence of two outcomes was observed, repetition $(31.5 \%)$ of self-inflicted violence and death records (43.8\%). Conclusion: The outlining of the epidemiological profile, in the southern region of Brazil, identified groups of older people who need more attention in the actions of prevention and occurrence of self-inflicted violence, being male older people, younger and with low education.

\section{INTRODUÇÃo}

O envelhecimento é um processo dinâmico, individual, irreversível, que promove um declínio progressivo das funções fisiológicas que interferem na capacidade orgânica e funcional das pessoas idosas ${ }^{1}$. Essas alterações podem ser percebidas de maneira positiva ou negativa pela pessoa idosa, ou seja, o histórico de sua vida determinará comportamentos futuros, podendo para muitos culminar na violência autoprovocada ${ }^{2}$.

Assim, a violência autoprovocada pode ser compreendida como aquela que ocorre quando uma pessoa pratica a ação consciente de autodestruição, esta é subdividida em comportamento suicida e autoagressão. $\mathrm{O}$ primeiro inclui ideação suicida, tentativas de suicídio e os suicídios; enquanto a autoagressão engloba atos como a automutilação e agressões a si próprio, desde as formas leves até as severas ${ }^{3}$.

Portanto, entende-se por tentativas de suicídio o ato deliberado de tirar a própria vida, porém sem efetivá-lo ${ }^{4}$. Alguns autores consideram existir uma fronteira tênue entre a ideação, a tentativa e o suicídio, embora haja a necessidade de se compreender que a ideação e as tentativas podem alternar-se $\mathrm{s}^{5}$.

Tentar explicar as razões pelas quais uma pessoa comete violência autoprovocada ou deseja fazê-lo, provém de uma gama de elementos que influenciam esse comportamento, tais fatores são oriundos de situações pessoais, sociais, psicológicas, culturais, biológicas e ambientais ${ }^{6}$. De maneira geral, as pessoas que cometem violência autoprovocada, estão vivenciando um sentimento de impossibilidade na identificação de alternativas para a solução de seus conflitos $^{3}$. Cavalcante et al. ${ }^{7}$, referem que a população idosa apresenta os mesmos comportamentos que outras faixas etárias relacionados as dificuldades em solucionar seus conflitos, já que tais dificuldades são provenientes de diferentes fatores sociais ${ }^{8}$.

Estima-se que as tentativas de suicídio são até quatro vezes maiores que os suicídios para as pessoas com faixa etária de 60 anos ou mais, podendo alcançar a proporção limite de um ato por um óbito ${ }^{2}$. O risco de óbito aumenta na medida em que aumentam o número de tentativas de suicídio, bem como está associado a intervalos menores de tempo entre uma violência e outra?. 
Referente aos óbitos autoprovocados, uma média de 11 mil pessoas tiram a própria vida por ano no Brasil. A região Sul do Brasil, concentra 23\% dos casos de suicídio do território nacional ${ }^{10}$. A taxa por suicídio nessa mesma região no ano de 2012, para pessoas de 60 anos ou mais foi de 16 por 100.000 habitantes ${ }^{11}$. Em relação aos suicídios a ciência já quantificou números relacionados aos idosos, porém nota-se escassez de estudos que envolvam essa população quando se refere a tentativas de suicídio ${ }^{12}$.

Desta forma, as violências autoprovocadas em pessoas idosas são um problema social e de saúde pública, isto posto existe a necessidade imperiosa de investir em estudos sobre a temática, uma vez que há ausência de pesquisas publicizadas com essa população na região Sul do Brasil.

No Brasil, uma das possibilidades de estabelecer resultados epidemiológicos referente a violência autoprovocada, é utilizando o banco de dados nacional do sistema de Vigilância de Violência Interpessoal e Autoprovocada (VIVA/SINAN), o qual é alimentado pelas notificações realizadas pelos profissionais ou instituições de saúde ${ }^{12}$. Mais que apenas produzir dados quantificáveis sobre a violência autoprovocada, há a necessidade de conceder aos profissionais e autoridades de saúde o conhecimento sobre a realidade das pessoas idosas de forma contextualizada a fim de possibilitar a criação de novas políticas públicas ${ }^{13,14}$. Portanto, objetivamos descrever as características das pessoas idosas que cometeram violência autoprovocada, notificados no Sistema de Informação de Agravos de Notificação (SINAN) na região Sul do Brasil de 2009 a 2016.

\section{MÉTODO}

Trata-se de um estudo de abordagem quantitativo, descritivo, retrospectivo, com dados secundários. As notificações de violências autoprovocadas das pessoas idosas estudadas se concentram na região Sul do Brasil (Rio Grande do Sul, Santa Catarina e Paraná), no período de 01 de janeiro de 2009 a 31 de dezembro de 2016. O estatuto do Idoso ${ }^{15}$ considera pessoa idosa no Brasil, aquela que completa sessenta ou mais anos, portanto essa foi a população alvo deste estudo.
Segundo o Instituto Brasileiro de Geografia e Estatística ${ }^{16}$, a projeção da população idosa na região Sul do Brasil no ano de 2025 corresponderá a 6.047.338 pessoas. A perspectiva em porcentagem para a população idosa, referente ao ano citado, será de aproximadamente 18\% para os estados do Paraná e Santa Catarina e 22\% para o Rio Grande do Sul.

O período estudado foi delimitado de 2009 a 2016, em função das notificações de violência terem sido inseridas no SINAN no ano de 2009 e em 2016 devido a disponibilidade do banco de dados estar consolidado pelo Ministério da Saúde (MS). A seleção das variáveis presentes no estudo baseou-se nas Fichas de Notificação/Investigação Individual de Violência Doméstica, Sexual e/ou outras Violências, publicizada no ano de 2008 e Ficha de Notificação Individual Violência Interpessoal/Autoprovocada, publicada no ano de 2015. Considerou-se as alterações realizadas pelo MS no ano de 2015, optou-se pela escolha, na sua maioria, dos dados em comum nos dois modelos de notificação ${ }^{11}$. As variáveis selecionadas, das fichas, visaram responder aos objetivos do estudo.

Optou-se por separar as variáveis em características sociodemográficas (idade, sexo, cor da pele, escolaridade e situação conjugal/estado civil), localização (unidade federativa, zona de ocorrência, local da ocorrência e turno da ocorrência) e da violência (meio de agressão, violência de repetição e evolução), das pessoas idosas que cometeram violência autoprovocada.

$\mathrm{Na}$ ficha de notificação de 2008, estava presente o campo evolução que registrava o desfecho do caso, podendo, entre eles, haver o óbito decorrente da violência. As pesquisadoras entenderam ser relevante a inclusão desse dado no estudo, mesmo não estando incluído na ficha de 2015. Neste estudo, optouse por utilizar todas as notificações de violência autoprovoca pelas pessoas idosas presentes no banco de dados, mesmo que algumas não estejam totalmente preenchidas.

As variáveis foram extraídas do banco de dados nacional do sistema do SINAN entre novembro e dezembro de 2019 e submetidas à análise estatística descritiva, por meio de frequência simples e proporção (\%), estratificados por faixa etária (60-69 anos; 7079 anos; 80 anos ou mais) e realizado intervalos 
de confiança (IC95\%). A significância estatística foi testada usando-se o teste qui-quadrado $\left(\chi^{2}\right)$ e considerado o valor de $p \leq 0,05$.

O banco de dados foi solicitado por pesquisadora do grupo de pesquisa em Violência e Saúde da Universidade Federal de Santa Catariana através do sistema de acesso à informação, e cedido pela Secretaria de Vigilância em Saúde do Ministério da Saúde, após assinatura do termo de responsabilidade de cessão das bases de dados da secretaria de vigilância em saúde, com aval da Coordenação Geral de Vigilância de Agravos e Doenças Não Transmissíveis. Quanto os cuidados éticos, a pesquisa utilizou dados secundários, portanto não precisou ser submetida ao comitê de ética devido a resolução do Conselho Nacional de Saúde (CNS) 510/2016, artigo $1^{\circ}$, parágrafo único e alínea $\mathrm{V}$, refere que pesquisas com bancos de dados, cujas informações são agregadas, sem possibilidade de identificação individual não são registradas e passadas nos Comitês de Ética em Pesquisa/Comissão Nacional de Ética em Pesquisa (CEP/CONEP).

\section{RESULTADOS}

Foram analisadas 2.290 notificações do SINAN de violências autoprovocadas de pessoas idosas no Sul do Brasil entre 2009 e 2016. A média da idade das pessoas idosas foi 68,9 anos, sendo que a $43,9 \%$ são do sexo feminino e $56,1 \%$ do sexo masculino. Declararam-se como brancos $90,9 \%$ e $5,9 \%$ como pardos, destas $6,3 \%$ tinham de zero a quatro anos de estudo, seguido de $28,6 \%$ que tinham de cinco a oito anos de estudo; características essas que prevalecem nas três estratificações por idade. Declararam-se casados ou em união estável $54,0 \%$ e 23,0\% viúvos, nota-se que os casados predominam nas três faixas etárias estratificadas. Informações essas encontradas na tabela 1.

A tabela 2 descreve sobre as características dos locais onde ocorreu a violência autoprovocada das pessoas idosas, na região Sul do Brasil. O estado do Rio Grande do Sul registrou 50,7\% das notificações, seguido de Santa Catarina 27,1\% e por fim, o Paraná com $22,2 \%$, evidenciando que o estado do Rio Grande do Sul apresenta maior porcentagem de registros de violência autoprovocada nas três faixas etárias estratificadas. As violências autoprovocadas ocorreram predominantemente na zona urbana ou periurbana com $81,8 \%$ dos casos registrados. O local de maior ocorrência das violências autoprovocadas registradas foram a residência/ habitação coletiva com $90,2 \%$. O horário de ocorrência foi 33,3\% no período da manhã e no período da tarde com 33,1\%.

A tabela 3 refere sobre as características dos meios de agressão utilizados pelas pessoas idosas nas violências autoprovocadas, sendo que o enforcamento com $29,9 \%$, o envenenamento $24,9 \%$, o objeto perfuro cortante $11,5 \%$, a força corporal/espancamento $6,4 \%$ e a arma de fogo $6,2 \%$. Registrou-se que $31,5 \%$ dos casos de violência autoprovocada se repetiram. Foram a óbito em decorrência das violências autoprovocadas 397 pessoas idosas (43,8\%), 203 (51,3\%) de 60 a 69 anos, $126(31,7 \%)$ de 70 a 79 anos e $68(17,1 \%)$ de 80 anos ou mais.

Tabela 1. Características sociodemográficas das pessoas idosas que cometeram violência autoprovocada, notificadas no SINAN. (N=2.290). Região Sul. Brasil. 2009-2016.

\begin{tabular}{|c|c|c|c|c|c|c|c|c|}
\hline \multirow{2}{*}{ Características } & \multirow{2}{*}{$\begin{array}{l}\text { Total } \\
\mathrm{N}(\%)\end{array}$} & \multicolumn{2}{|l|}{ 60-69 anos } & \multicolumn{2}{|l|}{ 70-79 anos } & \multicolumn{2}{|c|}{80 anos ou mais } & \multirow[t]{2}{*}{ Valor de $p$} \\
\hline & & $\mathrm{n}(\%)$ & IC 95\% & $\mathrm{n}(\%)$ & IC $95 \%$ & $\mathrm{n}(\%)$ & IC 95\% & \\
\hline $\operatorname{Sexo}(n=2.290)$ & & & & & & & & $<0,001$ \\
\hline Feminino & $1.005(43,9)$ & $666(66,3)$ & $63,2-69,1$ & $237(23,6)$ & $21,0-26,3$ & $102(10,1)$ & $8,4-12,1$ & \\
\hline Masculino & $1.285(56,1)$ & $738(57,4)$ & $54,7-60,1$ & $382(29,7)$ & $27,2-32,2$ & $165(12,9)$ & $11,1-14,7$ & \\
\hline Cor da pele $(n=2.229)$ & & & & & & & & 0,252 \\
\hline Branca & $2.026(90,9)$ & $1.231(60,8)$ & $58,6-62,8$ & $561(27,7)$ & $25,7-29,6$ & $234(11,5)$ & $10,2-13,0$ & \\
\hline Preta & $58(2,6)$ & $33(56,9)$ & $43,5-69,2$ & $17(29,3)$ & $18,8-42,6$ & $8(13,8)$ & $06,9-25,6$ & \\
\hline Amarela/Indígena & $13(0,6)$ & $8(61,5)$ & $30,5-85,3$ & $3(23,1)$ & $6,3-57,1$ & $9(15,4)$ & $3,0-50,9$ & \\
\hline Parda & $132(5,9)$ & $94(71,2)$ & $62,8-78,3$ & $23(17,4)$ & $11,7-24,9$ & $15(11,4)$ & $6,9-18,1$ & \\
\hline
\end{tabular}


Continuação da Tabela 1

\begin{tabular}{|c|c|c|c|c|c|c|c|c|}
\hline \multirow{2}{*}{ Características } & \multirow{2}{*}{$\frac{\text { Total }}{\mathrm{N}(\%)}$} & \multicolumn{2}{|l|}{ 60-69 anos } & \multicolumn{2}{|l|}{ 70-79 anos } & \multicolumn{2}{|c|}{80 anos ou mais } & \multirow[t]{2}{*}{ Valor de $p$} \\
\hline & & $\mathrm{n}(\%)$ & IC $95 \%$ & $\mathrm{n}(\%)$ & IC $95 \%$ & $\mathrm{n}(\%)$ & IC 95\% & \\
\hline $\begin{array}{l}\text { Escolaridade (anos) } \\
(\mathrm{n}=1.519)\end{array}$ & & & & & & & & $<0,001$ \\
\hline $0-4$ & $855(56,3)$ & $493(57,7)$ & $54,3-60,9$ & $253(29,6)$ & $26,6-32,7$ & $109(12,7)$ & $10,6-15,1$ & \\
\hline $5-8$ & $434(28,6)$ & $304(70,1)$ & $65,5-74,1$ & $99(22,8)$ & $19,0-27,0$ & $31(7,1)$ & $5,0-9,9$ & \\
\hline $9-11$ & $171(11,2)$ & $128(74,9)$ & $67,7-80,8$ & $30(17,5)$ & $12,5-24,0$ & $13(7,6)$ & $4,4-12,7$ & \\
\hline 12 ou mais & $59(3,9)$ & $508(4,7)$ & $72,7-92,0$ & $8(13,6)$ & $6,7-25,2$ & $1(1,7)$ & $0,2-11,6$ & \\
\hline Estado Civil $(\mathrm{n}=1.970)$ & & & & & & & & $<0,001$ \\
\hline Solteira & $241(12,2)$ & $158(65,6)$ & $59,2-71,3$ & $57(23,6)$ & $18,6-29,4$ & $26(10,8)$ & $7,4-15,4$ & \\
\hline Casada(o)/uni. Estável & $1.063(54,0)$ & $703(66,1)$ & $63,2-68,9$ & $273(25,7)$ & $23,1-28,3$ & $87(8,2)$ & $6,6-9,9$ & \\
\hline Viúva(o) & $453(23,0)$ & $177(39,1)$ & $34,6-43,6$ & $160(35,3)$ & $31,0-39,8$ & $116(25,6)$ & $27,1-29,8$ & \\
\hline Separada(o) & $213(10,8)$ & $163(76,5)$ & $70,3-81,7$ & $41(19,3)$ & $14,4-25,1$ & $9(4,2)$ & $2,2-7,9$ & \\
\hline
\end{tabular}

Fonte: VIVA/SINAN, 2009-2016.

Tabela 2. Características dos locais onde a violência autoprovocadas foi cometida pelas pessoas idosas notificadas no SINAN. (N=2.290). Região Sul. Brasil. 2009-2016.

\begin{tabular}{|c|c|c|c|c|c|c|c|c|}
\hline \multirow[t]{2}{*}{ Características } & \multirow{2}{*}{$\begin{array}{l}\text { Total } \\
\mathrm{N}(\%)\end{array}$} & \multicolumn{2}{|l|}{ 60-69 anos } & \multicolumn{2}{|l|}{ 70-79 anos } & \multicolumn{2}{|c|}{80 anos ou mais } & \multirow{2}{*}{$\begin{array}{l}\text { Valor } \\
\text { de } p\end{array}$} \\
\hline & & $\mathrm{n}(\%)$ & IC 95\% & $\mathrm{n}(\%)$ & IC 95\% & $\mathrm{n}(\%)$ & IC 95\% & \\
\hline Estado $(n=2.290)$ & & & & & & & & $<0,001$ \\
\hline Paraná & $509(22,2)$ & $323(63,5)$ & $59,1-67,5$ & $144(28,3)$ & $24,5-32,3$ & $42(8,2)$ & $6,1-10,9$ & \\
\hline Santa Catarina & $620(27,1)$ & $417(67,3)$ & $63,4-70,8$ & $146(23,5)$ & $20,3-27,0$ & $57(9,2)$ & $7,1-11,7$ & \\
\hline Rio Grande do Sul & $1.161(50,7)$ & $664(57,2)$ & $54,3-60,0$ & $329(28,3)$ & $25,8-31,0$ & $267(14,5)$ & $12,5-16,6$ & \\
\hline $\begin{array}{l}\text { Zona de ocorrência } \\
(\mathrm{n}=2.163)\end{array}$ & & & & & & & & 0,023 \\
\hline Urbana/periurbana & $1.769(81,8)$ & $1.105(62,5)$ & $60,1-64,6$ & $470(26,6)$ & $24,5-28,6$ & $194(10,9)$ & $9,5-12,5$ & \\
\hline Rural & $394(18,2)$ & $217(55,1)$ & $50,1-59,9$ & $123(31,2)$ & $26,8-35,9$ & $54(13,7)$ & $10,6-17,4$ & \\
\hline $\begin{array}{l}\text { Local da ocorrência } \\
(\mathrm{n}=2.234)\end{array}$ & & & & & & & & 0,064 \\
\hline $\begin{array}{l}\text { Residência/habitação } \\
\text { coletiva }\end{array}$ & $2.016(90,2)$ & $1.226(60,8)$ & $58,6-62,9$ & $545(27,0)$ & $25,1-29,0$ & $245(12,2)$ & $10,7-13,6$ & \\
\hline Via Pública & $106(4,8)$ & $74(69,8)$ & $60,2-77,8$ & $25(23,6)$ & $16,3-32,7$ & $7(6,6)$ & $3,1-13,3$ & \\
\hline Outros & $112(5,0)$ & $74(66,0)$ & $56,6-74,3$ & $32(28,6)$ & $20,8-37,7$ & $6(5,4)$ & $2,3-11,5$ & \\
\hline $\begin{array}{l}\text { Hora da ocorrência } \\
(n=1.490)\end{array}$ & & & & & & & & 0,086 \\
\hline Manhã & $491(33,0)$ & $276(56,2)$ & $51,7-60,5$ & $149(30,4)$ & $26,4-34,5$ & $66(13,4)$ & $10,6-16,7$ & \\
\hline Tarde & $493(33,1)$ & $299(60,6)$ & $56,2-64,8$ & $138(28,0)$ & $24,1-32,1$ & $56(11,4)$ & $8,8-14,4$ & \\
\hline Noite & $361(24,2)$ & $236(65,4)$ & $60,2-70,1$ & $85(23,5)$ & $19,4-28,2$ & $40(11,1)$ & $8,2-14,7$ & \\
\hline Madrugada & $145(9,7)$ & $98(67,6)$ & $59,4-74,7$ & $35(24,1)$ & $17,7-31,8$ & $12(8,3)$ & $4,7-14,0$ & \\
\hline
\end{tabular}

Fonte: VIVA/SINAN, 2009-2016. 
Tabela 3. Características dos meios de agressão das violências autoprovocadas cometidas pelas pessoas idosas notificadas no SINAN. (N=2.290). Região Sul. Brasil. 2009-2016.

\begin{tabular}{|c|c|c|c|c|c|c|c|c|}
\hline \multirow{2}{*}{ Características } & \multirow{2}{*}{$\begin{array}{l}\text { Total } \\
\mathrm{N}(\%) \\
\end{array}$} & \multicolumn{2}{|l|}{ 60-69 anos } & \multicolumn{2}{|l|}{ 70-79 anos } & \multicolumn{2}{|c|}{80 anos ou mais } & \multirow{2}{*}{$\begin{array}{l}\text { Valor } \\
\text { de } p\end{array}$} \\
\hline & & $\mathrm{n}(\%)$ & IC 95\% & $\mathrm{n}(\%)$ & IC 95\% & $\mathrm{n}(\%)$ & IC 95\% & \\
\hline \multicolumn{9}{|l|}{$\begin{array}{l}\text { Meio da } \\
\text { autoagressão }\end{array}$} \\
\hline \multicolumn{8}{|l|}{$\begin{array}{l}\text { Força corporal/ } \\
\text { espancamento } \\
(\mathrm{n}=2.220)\end{array}$} & \multirow[t]{3}{*}{0,860} \\
\hline Sim & $143(6,4)$ & $91(63,6)$ & $55,3-71,1$ & $36(25,2)$ & $18,6-33,0$ & $16(11,2)$ & $6,9-17,5$ & \\
\hline Não & $2.077(93,6)$ & $1.274(61,3)$ & $59,2-63,4$ & $559(26,9)$ & $25,0-28,8$ & $244(11,8)$ & $10,4-13,2$ & \\
\hline \multicolumn{9}{|l|}{$\begin{array}{l}\text { Enforcamento } \\
(\mathrm{n}=2.236)\end{array}$} \\
\hline Sim & $669(29,9)$ & $383(57,2)$ & $53,4-60,9$ & $191(28,6)$ & $25,2-32,1$ & $95(14,2)$ & $11,7-17,0$ & \multirow[t]{2}{*}{0,014} \\
\hline Não & $1.567(70,1)$ & $990(63,2)$ & $60,7-65,5$ & $409(26,1)$ & $23,9-28,3$ & $168(10,7)$ & $9,2-12,3$ & \\
\hline \multicolumn{9}{|c|}{$\begin{array}{l}\text { Objeto contundente } \\
(\mathrm{n}=2.224)\end{array}$} \\
\hline $\operatorname{Sim}$ & $36(1,6)$ & $21(58,3)$ & $41,1-73,7$ & $8(22,2)$ & $11,1-39,4$ & $7(19,5)$ & $9,2-36,4$ & \multirow[t]{2}{*}{0,328} \\
\hline Não & $2.188(98,4)$ & $1.347(61,6)$ & $59,5-63,5$ & $588(26,9)$ & $25,0-28,7$ & $253(11,6)$ & $10,2-12,9$ & \\
\hline \multicolumn{9}{|c|}{$\begin{array}{l}\text { Objeto perfuro } \\
\text { cortante }(n=2.229)\end{array}$} \\
\hline $\operatorname{Sim}$ & $257(11,5)$ & $145(56,4)$ & $50,2-62,3$ & $84(32,7)$ & $27,1-38,6$ & $28(10,9)$ & $7,6-15,3$ & \multirow[t]{2}{*}{0,089} \\
\hline Não & $1.972(88,5)$ & $1.223(62,0)$ & $59,8-64,1$ & $517(26,2)$ & $24,3-28,2$ & $232(11,8)$ & $10,4-13,2$ & \\
\hline \multicolumn{9}{|c|}{$\begin{array}{l}\text { Substância/ objeto } \\
\text { quente }(n=2.222)\end{array}$} \\
\hline Sim & $31(1,4)$ & $19(61,3)$ & $42,4-77,2$ & $7(22,6)$ & $10,6-41,5$ & $5(16,1)$ & $6,5-34,6$ & \multirow[t]{2}{*}{0,691} \\
\hline Não & $2.191(98,6)$ & $1.347(61,5)$ & $59,4-63,4$ & $589(26,9)$ & $25,0-28,7$ & $255(11,6)$ & $10,3-13,0$ & \\
\hline \multicolumn{8}{|c|}{$\begin{array}{l}\text { Envenenamento } \\
(\mathrm{n}=2.222)\end{array}$} & \multirow[t]{3}{*}{$<0,001$} \\
\hline Sim & $553(24,9)$ & $406(73,4)$ & $69,5-76,9$ & $118(21,3)$ & $18,1-24,9$ & $29(5,3)$ & $3,6-7,4$ & \\
\hline Não & $1.669(75,1)$ & $960(57,5)$ & $55,1-59,8$ & $478(28,7)$ & $26,5-30,8$ & $231(13,8)$ & $12,2-15,5$ & \\
\hline \multicolumn{9}{|l|}{$\begin{array}{l}\text { Arma de fogo } \\
(n=2.230)\end{array}$} \\
\hline Sim & $138(6,2)$ & $61(44,2)$ & $36,0-52,6$ & $57(41,3)$ & $33,3-49,7$ & $20(14,5)$ & $9,4-21,5$ & \multirow[t]{2}{*}{$<0,001$} \\
\hline Não & $2.092(93,8)$ & $1.309(62,6)$ & $60,4-64,6$ & $543(25,9)$ & $24,1-27,8$ & $240(11,5)$ & $10,1-12,9$ & \\
\hline \multicolumn{8}{|c|}{ Repetição (n=1.812) } & \multirow{3}{*}{0,081} \\
\hline $\operatorname{Sim}$ & $571(31,5)$ & $376(65,9)$ & $61,8-69,6$ & $136(23,8)$ & $20,4-27,4$ & $59(10,3)$ & $8,0-13,1$ & \\
\hline Não & $1.241(68,5)$ & $749(60,4)$ & $57,5-63,0$ & $345(27,8)$ & $25,3-30,3$ & $147(11,8)$ & $10,1-13,7$ & \\
\hline \multicolumn{9}{|l|}{ Evolução* } \\
\hline \multicolumn{9}{|l|}{ Óbito (n=906) } \\
\hline $\operatorname{Sim}$ & $397(43,8)$ & $203(51,3)$ & $46,2-56,0$ & $126(31,7)$ & $27,3-36,5$ & $68(17,1)$ & $13,7-21,1$ & \multirow[t]{2}{*}{$<0,001$} \\
\hline Não & $509(56,2)$ & $325(63,8)$ & $59,5-67,9$ & $130(25,5)$ & $21,9-29,5$ & $54(10,6)$ & $8,2-13,6$ & \\
\hline
\end{tabular}

*presentes nas notificações até junho de 2015; Fonte: VIVA/SINAN, 2009-2016. 


\section{DISCUSSÃO}

Neste estudo, caracterizou-se o perfil sociodemográfico das pessoas idosas que cometeram violência autoprovocada notificados no SINAN na região Sul do Brasil, os principais resultados evidenciam que a faixa etária predominante foi 60 69 anos, do gênero masculino, pessoas de cor de pele branca, baixo nível de escolaridade e casadas.

Neste contexto, a faixa etária de maior ocorrência das violências autoprovocadas das pessoas idosas, demonstrou ser no início do ciclo do envelhecimento. A literatura ${ }^{17}$ enfatiza que a medida que a idade avança aumenta a letalidade das tentativas de suicídio, já que o processo de envelhecimento é multifatorial, promovendo alterações anatômicas e funcionais no organismo ${ }^{18}$, naturalmente, ao avançar da idade o corpo está mais frágil e suscetível as ações externas.

Referente ao gênero, na região Sul do Brasil, o indivíduo do sexo masculino foi o principal autor das violências autoprovocadas, um estudo realizado em São Paulo, Capital, encontrou um dado semelhante ${ }^{19}$, porém, outras pesquisas evidenciaram que as mulheres na faixa etária estudada apresentam maiores tentativas em tirar a própria vida que os homens ${ }^{17-22}$.

As hierarquias de poder social, entre os sexos seguem as demandas do patriarcado, ou seja, um modelo que destaca a autoridade dos homens sobre as mulheres e os filhos ${ }^{23}$. As mulheres idosas foram encaminhadas num processo a não perceberem suas necessidades básicas, todavia suas obrigações versavam em prol de cuidar do outro, quer seja marido, filhos ou familiares. A masculinidade e virilidade são conflitos aterradores para os homens idosos, quando esses se afastam do trabalho, da posição social familiar de provedores que é imposta pelo poderio hierárquico social. Nessa linha de pensamento, é que as pessoas idosas do sexo masculino são as vítimas desse modelo social, que não permite e não perdoa demonstrar vulnerabilidade ${ }^{24}$.

No último Censo do IBGE ${ }^{25}, 78,3 \%$ da população da região Sul do Brasil se autodeclarou de cor branca, seguido pela parda $16,7 \%$. Informação essa que pode ter relação com o resultado deste estudo, pois o número de notificações foi predominantemente em pessoas brancas $(91,0 \%)$ e pardas $(5,9 \%)$. Outros estudos brasileiros que abordam a temática da violência autoprovocada em pessoas idosas, referiram que as vítimas eram de raça/cor pardas seguidas pelas brancas ${ }^{18,26,27}$. Sabe-se que os processos da identificação de cor de pele são subjetivos, complexos, multifacetados, que envolvem fatores históricos e socioculturais ${ }^{28}$. Além desses fatores, a maior concentração de brancos na região Sul é explicada pelo processo de colonização dessa região. Colonizada principalmente por imigrantes alemães, italianos e poloneses, na região Sul, as características fenotípicas e as preferências culturais dos habitantes aproximamse muito dos padrões europeus, inclusive no que se refere à cor da pele, predominantemente, branca ${ }^{29}$.

Quanto ao aspecto da escolarização esse é um importante fator para mensurar características de uma sociedade. O nível escolar da população estudada, predominou a baixa escolaridade, informação essa que corrobora com a literatura nacional e internacional $1^{19,26,27,30}$. A baixa escolaridade das pessoas idosas aumenta a probabilidade de um nível socioeconômico baixo, o que pode prejudicar a vida individual, familiar e social, podendo ser fator desencadeante da violência autoprovocada, já que dificulta um envelhecimento com dignidade ${ }^{-31}$.

Pesquisas realizadas na Turquia e Colômbia com pessoas idosas corroboram com os dados epidemiológicos deste estudo, os quais pontuaram que a maioria das violências autoprovocadas, não fatais, ocorreram com pessoas casadas ou que viviam em uma união estável ${ }^{22,32}$. Entendendo o aspecto cultural da região Sul do Brasil se faz necessário pautar que as pessoas idosas ao se casarem assumiam um compromisso de caráter indissolúvel, uma decisão assumida para toda a vida ${ }^{30}$.

Referente as características da violência, o estado do Rio Grande de Sul foi o que apresentou o maior número de registros de notificações por violência autoprovocada. Um estudo realizado no referido estado, evidenciou um aumento anual e gradativo dos casos de violência autoprovocada na população estudada, sendo 85/100.00 em 2005 para 149/100.000 em $2013^{33}$. Quando a violência autoprovocada resulta em morte nas pessoas idosas, o Sul do Brasil é a região que apresenta as maiores taxas, especialmente, o Rio Grande do Sul ${ }^{14,34}$. 
Referente ao local, a maior incidência das ocorrências se deu na zona urbana, demais pesquisadores encontraram achados equivalentes ${ }^{26}$. Entende-se esse dado, quando observa-se a concentração populacional brasileira, já que o maior número de pessoas reside na zona urbana ${ }^{25}$. Agregado a esse fato, sabe-se que nos centros urbanos ocorre a concentração dos serviços de saúde ${ }^{35}$. Outra informação relevante, a respeito do local, é que as violências autoprovocadas ocorreram predominantemente na residência/habitação coletiva, corroborando com outros estudos ${ }^{18,22,27}$.

Quanto ao meio de agressão utilizado para a violência autoprovocada, o enforcamento foi o escolhido pela maioria da amostra em questão, há também estudos sobre suicídio das pessoas idosas referindo ser esse o método mais utilizado ${ }^{21,27}$. Nessa perspectiva observa-se que o meio de agressão mais utilizado produz desfechos distintos.

Foi possível encontrar desfechos importantes neste estudo através das notificações: a alta porcentagem de repetição dos casos de violência autoprovocada em pessoas idosas e os 397 óbitos. Essa última informação constava na ficha de notificação até o ano de 2015, se o campo fosse mantido, na ficha atual, o valor poderia ser maior.

Uma limitação encontrada neste estudo, decorre da provável subnotificação dos casos de violência autoprovocadas, já que muitos casos não chegam aos serviços de saúde para um atendimento adequado, em função de a violência autoprovocada ser um tabu para a sociedade, família da vítima e a própria vítima ${ }^{34}$.

Trata-se de um trabalho descritivo, que não propõem demonstrar associações entre as variáveis das pessoas idosas que cometeram violência autoprovocada na região Sul do Brasil. A capacidade em determinar possíveis interferências causais e de extrapolar os resultados para outras populações é limitado.

\section{CONCLUSÃO}

O estudo apresentou dados que, em sua maioria, corroboram com pesquisas internacionais e nacionais sobre violência autoprovocada com pessoas idosas, portanto, nota-se que há semelhança no perfil das vítimas, dos locais e dos meios de agressão utilizados. Com perfil epidemiológico delineado, torna-se possível identificar precocemente os fatores de risco e, desta forma, evitar que tentativas de violência autoprovocadas ocorram.

Os dados aqui apresentados impelem que políticas públicas de prevenção e promoção de violências autoprovocadas sejam efetivadas dentro do Sistema Único de Saúde e da rede responsável pelos cuidados das pessoas idosas. Sugerem-se novas pesquisas referentes à temática para que ocorram aprofundamentos sobre as violências autoprovocadas, não fatais, cometidas pelas pessoas idosas, visto que este assunto ainda carece de estudos.

Editado por: Tamires Carneiro de Oliveira Mendes

\section{REFERÊNCIAS}

1. de Sousa ANV, Marquette FR. Envelhecimento da população brasileira: avanço legislativo e o compromisso social. Rev Univap. 2018;24(45):3447. Disponível em: http://dx.doi.org/10.18066/ revistaunivap.v24i45.1996 .

2. Figueiredo AEB, da Silva RM, Vieira LJES, Mangas RMN, de Sousa GS, Freitas JS, et al. É possível superar ideações e tentativas de suicídio? Um estudo sobre idosos. Ciênc Saúde Colet. 2015;20(6):1711-9. Disponível em: http:// www.scielosp.org/scielo.php?script=sci_ arttext\&pid=S1413-81232015000601711

3. Organização Mundial da Saúde. Relatório Mundial sobre Violência e Saúde. Genebra: OMS; 2002.

4. Brasil. Ministério da Saúde. Política Nacional de Humanização da Atenção e Gestão do SUS. Acolhimento e classificação de risco nos serviços de urgência. Brasília, DF: MS; 2009.

5. Teixeira SMO, Souza LEC, Viana LMM. O suicídio como questão de saúde pública. Rev Bras Promoç Saúde. 2018;31(3):1-3. Disponível em: http:// periodicos.unifor.br/RBPS/article/view/8565. 
6. World Health Organization. Preventing suicide: a global imperative. Genève: WHO; 2014.

7. Cavalcante FG, Minayo MCS, Mangas RMN. Diferentes faces da depressão no suicídio em idosos. Ciênc Saúde Colet. 2013;18(10):2985-94. Disponível em: https://doi.org/10.1590/S1413-81232013001000023 .

8. Mellqvist M, Wiktorsson S, Joas E, Ostling S, Skoog I, Waern M. Sense of coherence in elderly suicide attempters: the impact of social and health-related factors. Int Psychogeriatr. 2011;23(6):986-93. Disponível em: https://doi.org/10.1017/S1041610211000196 .

9. Zhang W, Ding H, Su P, Xu Q, Du L, Xie C, et al. Prevalence and risk factors for attempted suicide in the elderly: a cross-sectional study in Shanghai, China. Int Psychogeriatr. 2016;29(5):709-15. Disponível em: http://doi.org/10.1017/S1041610216002283 .

10. Brasil. Ministério da Saúde. Perfil epidemiológico das tentativas e óbitos por suicídio no Brasil e a rede de atenção à saúde. Brasília, DF: MS; 2017.

11. Machado DB, dos Santos DN. Suicídio no Brasil, de 2000 a 2012. J Bras Psiquiatr. 2015;64(1):45-54. Disponível em: https://doi.org/10.1590/00472085000000056 .

12. Brasil. Ministério da Saúde. Viva: instrutivo notificação de violência interpessoal e autoprovocada. Brasília, DF: MS; 2016.

13. Grigoletto AP, Souto VT, Terra MG, Tisott ZL, Ferreira CN. Tentativas de suicídio notificadas em um hospital de ensino no Estado do Rio Grande do Sul, 2014-2016. Rev Pesqui. 2020;12:447-53. Disponível em: http://seer.unirio.br/index.php/ cuidadofundamental/article/viewFile/8349/pdf_1 .

14. Cabral DVS, Pendloski J. Mortalidade por suicídio em idosos: uma análise do perfil epidemiológico no sul do Brasil. Rev Uningá. 2016;47(2):19-24.

15. Brasil. Lei no. 10.741, de 01 de outubro de 2003. Dispõe sobre o Estatuto do Idoso e dá outras providências. 2003. Disponível em: http://www. planalto.gov.br/ccivil_03/leis/2003/110.741.htm .

16. Instituto Brasileiro de Geografia e Estatística. Projeções e estimativas da população do Brasil e das Unidades da Federação [Internet]. Rio de Janeiro: IBGE; 2018. Disponível em: https://www.ibge.gov. br/estatisticas/sociais/populacao/9109-projecao-dapopulacao.html? $=\& \mathrm{t}=\mathrm{O}$-que-e .

17. Schmutte T, Olfson M, Xie M, Marcus SC. Deliberate self-harm in older adults: A national analysis of US emergency department visits and follow-up care. Int J Geriatr Psychiatry. 2019;34(7):1058-69. Disponível em: https://doi.org/10.1002/gps.5109 .
18. de Oliveira JMB, Vera I, Lucchese R, Silva GC, Tomé EM, Elias RA. Aging, mental health, and suicide. Envelhecimento, saúde mental e suicídio. Revisão integrativa. Rev Bras Geriatr Gerontol. 2018;21(4):488-98. Disponível em: http://www.scielo.br/scielo.php?script $=$ sci_ arttext\&pid=S1809-98232018000400488

19. Armond JE, Armond RE, Pereira TC, Chinaia C, Vendramini TL, Rodrigues CL. Self-injury and suicide attempt among the elderly population in the city of São Paulo. J Bras Psiquiatr. 2017;66(2):838. Disponível em: https://doi.org/10.1590/00472085000000154 .

20. Aravena JM, Gajardo J, Saguez R. Salud mental de hombres mayores en Chile: una realidad por priorizar. Rev Panam Salud Pública. 2019;42:1-1. Disponível em: https://scielosp.org/article/rpsp/2018.v42/e121/ .

21. Corona MB, Alfonso SK, Cuéllar LL, Hernández SM, Serra LS. Caracterización de la Conducta suicida en Cuba, 2011-2014. Rev Haban Cienc Méd. 2017;16(4):612-24. Disponível em: http://scielo. sld.cu/scielo.php?script=sci_arttext\&pid=S1729519X2017000400013.

22. Gokcellia DK, Tasar PT, Akcama NO, Sahinb $\mathrm{S}$, Akarcac FK, Aktasd KO, et al. Evaluation of attempted older adults suicides admitted to a University Hospital Emergency Department: Izmir study. Asian J Psychiatr. 2017;30:196-99. Disponivel em: https://doi.org/10.1016/j.ajp.2017.10.002 .

23. Figueiredo WS. Assistência à saúde dos homens: um desafio para os serviços de atenção primária. Ciênc Saúde Colet. 2005;10(1):105-9. Disponível em: https://doi.org/10.1590/S1413-81232005000100017 .

24. Meneghel SM, Gutierrez DMD, Silva RMda, Grubits S, Hesler LZ, Ceccon RF. Suicídio de idosos sob a perspectiva de gênero. Ciênc Saúde Colet. 2012;17(8):1983-92. Disponível em: https://doi. org/10.1590/S1413-81232012000800009 .

25. Instituto Brasileiro de Geografia e Estatística. Censo Brasileiro de 2010. Rio de Janeiro: IBGE; 2012.

26. Vilela AP, da Silva WP. A intoxicação exógena como método nas tentativas e suicídio entre os idosos. Rev Saúde. 2018;12(1):33-40. Disponível em: http://dx.doi. org/10.33947/1982-3282-v12n1-2-3569 .

27. Gomes AV, Cardoso PKB, Rocha FCV, Carvalho CMS, Sales MCV. Perfil sociodemográfico de idosos vítimas de suicídio em um estado do Nordeste do Brasil. Rev Baiana Enferm. 2018;32:1-9. Disponível em: http://dx.doi.org/10.18471/rbe.v32.26078 . 
28. Kabad J F, Bastos JL, Santos RV. Raça, cor e etnia em estudos epidemiológicos sobre populações brasileiras: revisão sistemática na base PubMed. Physis. 2012; 22(3):895-918. Disponível em: https:// doi.org/10.1590/S0103-73312012000300004 .

29. Proença BT, Soligo AF. O não-lugar do sujeito negro na educação brasileira. Rev Iberoam Educ. 2015;68(2):31-48. Disponível em: https://doi. org/10.35362/rie682183 .

30. Barceló JS, Cruz JP, Traba BP, Iglesias JMC, González JB, Álvarez RM. Perfil de los intentos de suicídio em personas mayores de 65 años del área sanitária de Santiago de compostela em el período de 2015 a 2017. Inf Psiquiátr. 2018;(235):89-107.

31. Seleghim MR, Bellasalma ACM, Mathias TAF, de Oliveira MLF. Caracterização das tentativas de suicídio entre idosos. Cogitare Enferm. 2012;17(2):277-283.
32. Monak IAO, Agudelo SAF, Ortiz JOG. Caracterización sociodemográfica de los suicidas adultos mayores de 60 años: Bogotá 2003-2007. Rev Colomb Psiquiatr. 2013;42(1):56-64. Disponível em:http://doi.org/10.1016/j.rcp.2013.11.006 .

33. Conte M, Cruz CW, da Silva CG, de Castilho NRM, Nicolella ADR. Encontros ou Desencontros: histórias de idosos que tentaram suicídio e a Rede de Atenção Integral em Porto Alegre/RS, Brasil. Ciênc Saúde Colet. 2015;20(6):1741-49. Disponível em: https://doi. org/10.1590/1413-81232015206.02452015 .

34. Pinto LW, de Assis SG, Pires TO. Mortalidade por suicídio em pessoas com 60 anos ou mais nos municípios brasileiros no período de 1996 a 2007. Ciênc Saúde Colet. 2012;17(8):1963-72. Disponível em https://doi.org/10.1590/S1413-81232012000800007 .

35. Arruda NM, Maia AG, Alves LC. Desigualdade no acesso à saúde entre as áreas urbanas e rurais do Brasil: uma decomposição de fatores entre 1998 a 2008. Cad Saúde Pública. 2018;34(6):1-14. Disponível em: https://doi.org/10.1590/0102-311X00213816 